have had to be very different during 2020. One aspect of the new working model, has been the requirement to wear, 'Personal Protective Equipment.' (PPE). Seeing healthcare workers in full PPE is a new experience for most children and it became obvious, early on during the pandemic, that we needed to find ways to make PPE more,' child-friendly' to minimise anxiety as much as possible for children presenting to hospital and in particular to theatre.

Methods Discussion with colleagues working in the operating theatres at Great Ormond Street Hospital revealed various adaptations to make PPE more, 'child-friendly.' Some children were asked for feedback related to this. A PubMed literature search regarding PPE use in paediatric settings and also the wearing of face masks by children was also conducted. In addition to this, an internet search provided information from other NHS Trusts.

Results Inventive solutions such as cartoon characters on visors and drawing on them have been very well received. Videos about PPE were also helpful. In addition, the importance of non-verbal communication has become very apparent.

Discussion Creating, 'child-friendly,' PPE has been hugely beneficial to children presenting to hospital during the pandemic. It helps to allay their fears and increases their understanding of the current, challenging world that they are living in.

\section{SIGHT ++: PROTOTYPING A COMPUTER VISION GUIDED ASSISTIVE TECHNOLOGY}

${ }^{1}$ Sven Finlay, ${ }^{2}$ Sheena Visram, 'Gisli Georgsson, 'Yanru Chen, 'Songping Lin, 'Xingda Cen, ${ }^{3}$ Costas Stylianou, ${ }^{3}$ Chris Feltham, ${ }^{3}$ Phillippa Chick, ${ }^{1}$ Dean Mohamedally, ${ }^{4}$ Neil J Sebire, ${ }^{1}$ Emmanuel Letier. ${ }^{1}$ UCL Department of Computer Sciences; ${ }^{2}$ University College London; ${ }^{3}$ Intel; ${ }^{4}$ GOSH DRIVE

\subsection{6/archdischild-2020-gosh.49}

Introduction The visually impaired in society are amongst the most impacted by social isolation restrictions of the COVID19 pandemic, fueling research into assistive technologies, including devices primed for computer vision task-orientated image recognition. We present a Proof of Concept prototype modular system that uses Intel RealSense depth cameras connected to a modular ML inference platform to construct near field object information that guides and encourages exploration for users.

Methods Early engagement from experts in the field of global disability enabled us to better appreciate orientation, mobilityrelated considerations, sensory components and meaningful voice instructions. We subsequently designed a novel modular, extensible platform that runs inference classification and depth detection on camera input, then uses heuristic AI Priortiser to analyse and identify essential guidance output for the users.

Results Sight ++ uses object recognition to accurately inform users on near field objects, including data on proximity. By parsing the items through a series of environment rules, which results in objects having more or less relative importance, the system can output qualified audio guidance for obstacle avoidance and awareness. The use of OpenVINO resulted in a 2fold increase in performance of our inference classifiers. We anticipate that a miniaturised depth camera would be fitted to a backpack adjustable strap to offer real-time object recognition and meaningful notification of artefacts of interest at waist height and above.

Conclusion We have produced a robust, foundational assistive system which rather than replace recognised and trusted methods of navigation, introduces a new dimension of intelligence. Future versions will implement motion tracking of the objects, haptic feedback and a teleassistance function connecting a sighted volunteer to enhance guidance. We aspire that future improvements offer a seamless understanding of new environments and create a novel user experience for exploration that are designed for personalisability, social acceptability and social inclusion.

\section{SHAKE, RATTLE AND ROLL - A SERVICE EVALUATION OF A MULTIDISCIPLINARY MUSIC GROUP FOR FAMILIES WITH BERLIN HEARTS}

Zoe Vamplew, Sarah Rickard, Katya Herman, Emma Shkurka. Great Ormond Street Hospital

\subsection{6/archdischild-2020-gosh.50}

Introduction Ventricular assist devices are implanted as a bridge to heart transplant. Inpatient wait time on a Berlin Heart EXCOR (BH), can be up to 17 months. This prolonged hospitalisation has a significant impact on the child's psychological and physiological development, leading to limited educational and social integration. It is important that the developmental treatment of these children incorporates a family centred and holistic approach. This service evaluation assessed the effectiveness and safety of a multidisciplinary music group on $\mathrm{BH}$ patients, their carers and nursing staff.

Method A structured multidisciplinary music group was created by Physiotherapy, Music and Occupational therapy. Carer involvement was a key component. Each session included: singing, actions, turn taking, gross and fine motor activities and a story. Feedback via anonymous survey was sought from carers and nursing staff after six sessions regarding structure, content and environment.

Results Five children and six carers participated over 12 weeks. The average age was 21 months and wait time since implantation 126-368 days. All carers and three bedside nurses completed the survey.

$75 \%$ carers found the group beneficial and $75 \%$ felt their child enjoyed all components. Carers reported improved peer interaction and motor development. All advocated for the group to continue. Feedback included more actions and sensory stories. All nurses found the group overall 'extremely beneficial' and $66.67 \%$ felt the children 'extremely enjoyed' the sessions. $100 \%$ observed positive changes in the children and carers since commencement. No adverse events or safety concerns were raised.

Conclusion This service evaluation suggests that the multidisciplinary music group is a safe and valuable therapeutic component that can improve family experience and interaction for children on a $\mathrm{BH}$. Carers and staff observed a positive impact on motor and social development as well as enjoyment. The group is now routinely part of the $\mathrm{BH}$ therapy programme.

\section{POSCU - JOINED UP WORKING VIA GOSHLINK}

Bing Wang, Lynne Riley, Rachel Edmead, Joshua Giddings, Chris Grant. Great Ormond Street Hospital

\subsection{6/archdischild-2020-gosh.51}

All members of the patient's care team using GOSHLink can view the patient's full, contemporaneous electronic medical 